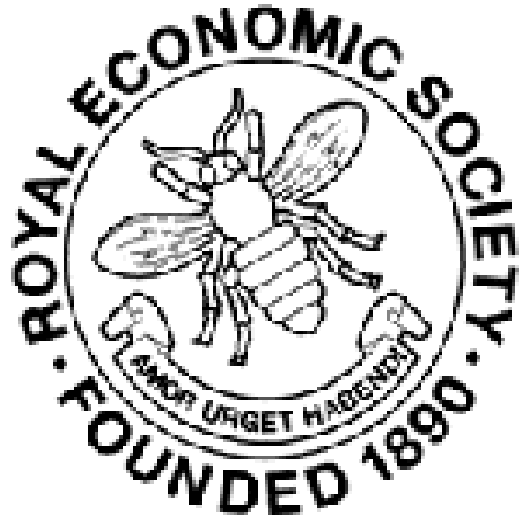

\title{
Recent Contributions to Mathematical Economics
}

\section{Author(s): C. P. Sanger}

Source: The Economic Journal, Vol. 5, No. 17 (Mar., 1895), pp. 113-128

Published by: Wiley on behalf of the Royal Economic Society

Stable URL: http://www.jstor.org/stable/2956239

Accessed: 27-06-2016 02:40 UTC

Your use of the JSTOR archive indicates your acceptance of the Terms \& Conditions of Use, available at

http://about.jstor.org/terms

JSTOR is a not-for-profit service that helps scholars, researchers, and students discover, use, and build upon a wide range of content in a trusted digital archive. We use information technology and tools to increase productivity and facilitate new forms of scholarship. For more information about JSTOR, please contact support@jstor.org.

Royal Economic Society, Wiley are collaborating with JSTOR to digitize, preserve and extend access to The Economic Journal 


\section{Recent Contributions to Mathematical Economics.}

ENGLISH students appear to have paid but scanty attention to a series of articles on the pure theory of political economy which have appeared during the past two or three years in the Giornale degli Economisti. The present writer attempts to place before the readers of this journal some of the more important parts of those articles in the hope that the inadequacy of the present paper may induce those who take a scientific interest in economics to read the original articles. The writer of the main series of articles entitled 'Considerazioni sui principii fondamentali dell' economia politica pura' 1 is Professor Pareto ; in addition to these he has also written several other artisles on special points which may be considered as subsidiary or supplementary to the main series. ${ }^{2}$ Signor Barone has also written several articles; of these the two most important ones are 'On Consumers' Rent' and 'Sul trattamento di quistioni dinamiche.' 3 Only in so far as these writings deal with utility will they be considered in the following paper, which aims merely at putting forth what is already contained in the writings of these Italian economists.

Various assumptions may be made concerning marginal utility which approximate more or less closely to actual conditions. In general it is true that the utility we obtain from the marginal increment of any commodity is a function of our personal character, our surroundings and the quantities of all the commodities that we possess. Non-mathematical text-books are, however, liable to assume, though perhaps not explicitly, that the utility a man derives from the marginal increment of any commodity is independent of the amounts of other commodities possessed by him. This is clearly very nearly true in many cases, very untrue in other cases. In respect to money it is very usual to assume that the marginal utility of money is nearly constant, and this, on the whole, seems to be a fair assumption. The value of Prof. Pareto's work chiefly consists in the fact that he makes his assumptions clear, and enables us to see what conclusions are obtainable from what premises. He never attempts to atone for the rashness of his assumptions by the looseness of his deductions. The most important part of Sig. Barone's work consists in showing that approximately true assumptions are sufficiently accurate for practical purposes.

The first proposition which will be quoted from Prof. Pareto is of

\footnotetext{
${ }^{1}$ In the Giornale degli Economisti, May, June, August, 1892 ; January, October 1893.

2 'On an error of Cournot's,' January, 1892. 'On Prof. Walras and Herrn Auspitz and Lieben,' March 1892. 'Teoria mathematica dei cambi forestieri,' February 1894. ' Il massimo di utilità dato dalla libera concorrenza,' July 1894. La legge della domanda, January 1895.

${ }^{3}$ September and October 1894.
}

No. 17.- - VOL. V 
some interest, as it shows us that, upon certain assumptions, which are frequently made, it is most unlikely that the marginal utility of money should remain theoretically constant. The next two are concerned with the connection between the laws of marginal utility and those of supply and demand. A failure of the solution in the particular case of two commodities leads Prof. Pareto to introduce the further consideration of saving. From the case of individuals he proceeds to that of a society, showing the connection between the marginal utility of any commodity for the society as a whole, and the marginal utilities for the separate individuals. Up to this point he has kept to the above-mentioned assumption about the marginal utility of any commodity. Still keeping to this assumption, he turns to the consideration of total utility, and gives a very interesting maximum problem. He then considers the more general assumption that the marginal utility of a commodity is a function of the amounts of all the commodities possessed, and the in teresting question arises, 'Does a total utility function, analogous to a potential function, exist?' And this suggests the further question, 'If this utility function does exist, what is its most probable form?' At present we generally answer the first of these questions in the affirmative, scarcely any attempt has yet been made to answer the second. In addition to the extracts from Prof. Pareto's articles on the fundamental principles of pure political economy, the main part of his article entitled 'Il massimo di utilità dato dalla libera concorrenza' is here added because it introduces some further considerations into the ordinary problem of maximising utility.

The value of Sig. Barone's work consists chiefly in the discussion of small quantities. He shows that the different assumptions made by Professor Walras and Professor Marshall only lead to small differences in the result, so that there is no practical disagreement. The main part of his article on Consumers' Rent and part of a more recent article will be given.

Suppose that the prices $p_{b}, p_{c}, \ldots$ of commodities $B, C \ldots$ are measured in terms of a commodity $A$ (money as a common measure of exchange), and that a quantity $q_{a}$ of $A$ will buy quantities $r_{b}, r_{c} \ldots$ of $B, C, \ldots$, whose prices remain constant during the buying of successive portions; then we have

$$
q_{a}=p_{b} r_{b}+p_{c} r_{b}+
$$

If we make the further supposition that the utility of the marginal increment of $B$ is a function of $r_{b}, \ldots$ and if we then denote the marginal utilities of $A, B, C \ldots$ by $\phi_{u}\left(q_{a}\right), \phi_{b}\left(r_{b}\right), \phi_{c}\left(r_{c}\right)$, we have the equations

$$
\phi_{a}\left(q_{a}\right)=\frac{1}{p_{b}} \phi_{b}\left(r_{b}\right)=\frac{1}{p} \phi_{c}\left(v_{c}\right)=: .
$$


If now $\phi_{a}\left(q_{a}\right)$ is constant and equal to $m$, we can find $r_{b}, r_{c} \ldots$ in terms of $p_{b}, p_{c}, \ldots$ and $m$, and can put

$$
r_{b}=\psi_{b}\left(m p_{b}\right) ; r_{c}=\psi_{c}\left(m p_{c}\right) ;
$$

Putting these values in (1) we have

$$
q_{a}=p_{b} \psi_{b}\left(m p_{b}\right)+p_{c} \psi_{c}\left(m p_{c}\right)+\ldots
$$

Since the $p$ s are independent of one another, $q_{a}$ cannot be constant unless $p_{b} \psi_{b}\left(m p_{b}\right) ; p_{c} \psi_{c}\left(m p_{c}\right) ; \ldots$ are constant.

Let these equal $A_{b}, A_{c}, \ldots$ then we have

$$
p_{b} r_{b}=A_{b} ; p_{c} r_{c}=A_{c} ; .
$$

If $q_{a}$ be not constant, then we can say that to definite values of $m$, $p_{b}, p_{e}, \ldots$ there corresponds a definite value of $q_{a}$, and vice versa.

The preceding equations combined with (1) give

$$
\phi_{b}\left(r_{b}\right)=m p_{b}=\frac{m A_{b}}{r_{b}} ; \phi_{c}\left(r_{c}\right)=m p_{c}=\frac{m A_{c}}{r_{c}} ; \ldots
$$

that is to say, the marginal utility of money will only remain theoretically constant in the case in which the marginal utilities of all the other commodities concerned obey Bernouilli's hypothesis. ${ }^{1}$

Notice that equations (4) are equivalent to saying that the elasticities of demand are equal to $1 .{ }^{2}$

We will postpone for the present any further discussion of the question of the marginal utility of money, and will turn to the important problem of determining the laws of supply and demand when the marginal utilities are given and conversely. Making the same assumption concerning the marginal utilities, and writing $r_{a}$ for $-q_{a}$, so that our problem becomes one of general exchange, we have as before:-

and

$$
r_{a}+p_{b} r_{b}+p_{c} r_{c}+\ldots=0 . . . . .
$$

$$
\phi_{a}\left(r_{a}\right)=\frac{1}{p_{b}} \phi_{b}\left(r_{b}\right)=\frac{1}{p_{c}} \phi_{c}\left(r_{c}\right)=. . . .
$$

If there are $n$ commodities, we have $n-1$ equations in (2), which, together with (1), give us $n$ equations, which enable us to express $r_{a}, r_{b} \ldots$ in terms of the $p \mathrm{~s}$ and $\phi \mathrm{s}$. Substituting from (2) in (1) we obtain

$$
r_{a} \phi_{a}\left(r_{a}\right)+r_{b} \phi_{b}\left(r_{b}\right)+r_{c} \phi_{c}\left(r_{c}\right)+\ldots=0 . . .
$$

From this we can obtain an equation in the form

$$
\phi_{a}\left(r_{a}\right)=\psi\left\{r_{b} \phi_{b}\left(r_{b}\right)+r_{c} \phi_{c}\left(r_{c}\right)+\ldots\right.
$$

and we therefore obtain

$$
p_{b}=\phi_{b}\left(r_{b}\right) / \psi\left\{r_{b} \phi_{b}\left(r_{b}\right)+r_{c} \phi_{c}\left(r_{c}\right)+\ldots\right\}
$$

1 Pareto, G. d. E., June 1892.

2 Prof. Pareto merely says that they must be constant, but this constant is clearly unity. 
which gives us the relation between $p_{b}$ and $r_{b}$ when $r_{c}, r_{d} \ldots$ are known. ${ }^{1}$

The converse problem: Given the laws of supply and demand, to determine the marginal utilities -introduces a fresh difficulty.

In the equations (1) and (2) above we took the $\overline{n-1} p$ s as independent variables: suppose now that we take $r_{b}, r_{c} \ldots$ as the $\bar{n}-1$ independent variables. The condition that $\phi_{b}$ is a function of $r_{b}$ alone is given by the $\overline{n-2}$ equations $\frac{\partial}{\partial r_{c}}\left(p_{b} \phi_{a}\right)=0 ; \frac{\partial}{\partial r_{d}}\left(p_{b} \phi_{a}\right)=0$; and since there are $\overline{n-1}$ of $\phi_{b}, \phi_{c}, \ldots$ we have $\overline{n-1} \cdot \overline{n-2}$ of these equations. From observation we obtain the $\overline{n-1}$ quantities, $\frac{\partial p_{b}}{\partial r_{b}}$, $\frac{\partial p_{c}}{\partial r_{c}}$, so that in all we have $(n-1)^{2}$ equations. The number of unknowns (the quantities $\phi_{a}{ }^{\prime}\left(r_{a}\right) \ldots$ ) is $n$; so that in general our $(n-1)^{2}$ equations are not independent. But in the case of two unknowns we have only 1 equation, so that the solution fails. To obtain a solution we will take account of the possibility of saving. Suppose that an amount $s$ of $A$ is saved, then in place of 1 we have :-

$$
r_{a}+p_{b} r_{b}+p_{c} r_{c}+\ldots=s .
$$

The number of our independent variables $\left(r_{a}, r_{b}, \ldots\right)$ is now $n$. The partial differential equations are now

$$
n(n-2)+n=n(n-1)
$$

in number, and as before we have $n$ unknowns. Hence, in the case of two commodities we have two equations to determine our two .unknowns. ${ }^{2}$

We proceed now to consider the case of a society of individuals. Let the prices of the commodities $A, B, C, \ldots$ be $1, p_{b}, p_{c}, \ldots$ and let the quantity of the commodity $C$, demanded by the individual $\mu$, be $r_{\mu c} \ldots$ and let his marginal utility of this be $\phi_{\mu c}\left(r_{\mu c}\right)$.

For the whole society let the quantities demanded be $r_{a}, r_{b}, r_{c} \ldots$, and the marginal utilities $\phi_{a}\left(r_{a}\right), \phi_{b}\left(r_{b}\right), \phi_{c}\left(r_{c}\right) \ldots$ We have, in the first place, the $n$ equations

$$
\begin{aligned}
& r_{a}=r_{1 a}+r_{2^{a}}+\ldots \\
& r_{b}=r_{1^{b}}+r_{2^{b}}+\ldots \\
& .
\end{aligned}
$$

If $n$ is the number of individuals, and $\theta$ the number of commodities, we have the $(n-1)(\theta-1)$ equations

$$
\begin{aligned}
& \left.\begin{array}{l}
\frac{\phi_{1 b}\left(r_{1 b}\right)}{\phi_{1 a}\left(r_{1 a}\right)}=\frac{\phi_{2 b}\left(r_{2 b}\right)}{\phi_{2 a}\left(r_{2 a}\right)}=\frac{\phi_{3 b}\left(r_{3 b}\right)}{\phi_{3 a}\left(r_{3 a}\right)}=\ldots \\
\frac{\phi_{1 c}\left(r_{1 c}\right)}{\phi_{1 a}\left(r_{1 a}\right)}=\frac{\phi_{2 c}\left(r_{2 c}\right)}{\phi_{2 a}\left(r_{2 a}\right)}-\frac{\phi_{3 c}\left(r_{3 c}\right)}{\phi_{3 a}\left(r_{3 a}\right)}=\ldots . \\
. \quad . \quad . \quad . \\
. \quad .
\end{array}\right\} . . \\
& 1 \text { Pareto, G. d. E., August } 1892 . \quad \text { Ibid. }
\end{aligned}
$$


There are two cases to be considered.

I. If there is no saving we have the $\theta$ equations

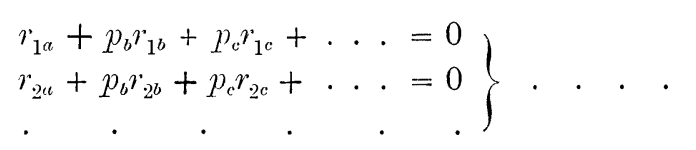

So that in all we have $n+(n-1)(\theta-1)+\theta=n \theta+1$ equations. Excluding, for the moment, one of these equations, we can determine the $n \theta$ unknowns $r_{1 e}, r_{2 c}, \ldots, r_{1 b}, r_{2 b}, \ldots$ with the remaining $n \theta$ equations in terms of $r_{u}, r_{b}, \ldots$

Putting these values in the equations

$$
p_{b}=\frac{\phi_{1 b}\left(r_{1 b}\right)}{\phi_{1 a}\left(r_{1 a}\right)} ; \ldots
$$

we obtain $p_{b}, p_{c} \ldots$ as functions of $r_{c}, r_{b}, \ldots$ but these will not necessarily be of the form

$$
p_{b}=\frac{\phi_{b}\left(r_{b}\right)}{\phi_{a}\left(r_{c}\right)} ; p_{c}=\frac{\phi_{c}\left(r_{c}\right)}{\phi_{a}\left(r_{c}\right)} ; \ldots
$$

and we have one equation over.

II. If we take saving into consideration, equations (3) fail. We may suppose that the $n$ quantities $r_{1 a}, r_{1 b}, r_{1 c}, \ldots$ are independent variables, and that $r_{2 a}, r_{3 k}, \ldots$ are functions of $r_{1 a}$, and so on. Then from equations (1) we see that in this case $r_{1 a}$ becomes a function of $r_{a} ; r_{1 b}$ of $r_{b}$, and so on; and putting these values in

we obtain

$$
p_{b}=\frac{\phi_{1 b}\left(r_{1 b}\right)}{\phi_{1 a}\left(r_{1 a}\right)} ; p_{c}=\frac{\phi_{1 c}\left(r_{1 c}\right)}{\phi_{1 a}\left(r_{1 a}\right)} ; \ldots
$$

$$
p_{b}=\frac{\phi_{b}\left(r_{b}\right)}{\phi_{a}\left(r_{a}\right)} ; p_{c}=\frac{\phi_{c}\left(r_{1 c}\right)}{\phi_{u}\left(r_{1 a}\right)} \ldots
$$

We proceeded as if instead of the equations (1) we had substituted the $(n-1)(\theta-1)+\theta-1$ equations

$$
\left.\begin{array}{rl}
\phi_{2 a}\left(r_{2 a}\right)=H_{2} \phi_{1 a}\left(r_{1 a}\right) ; & \phi_{3 a}=H_{3} \phi_{3 a} \\
\phi_{2 b}\left(r_{2 b}\right)=H_{2} \phi_{1 b}\left(r_{1 b}\right) ; & \phi_{3 b}=H_{3} \phi_{3 b} \\
\phi_{2 c}\left(r_{2 c}\right)=H_{2} \phi_{1 c}\left(r_{1 c}\right) ; \quad \phi_{3 c}=H_{3} \phi_{3 c}
\end{array}\right\}
$$

where $H_{2}, H_{3}$ are arbitrary constants. These equations, together with the $n$ equations (1) give us the $n \theta$ equations necessary to determine our $n \theta$ unknowns. ${ }^{1}$

On the same supposition as before - that the marginal utility of a. commodity is a function of the quantity of that commodity alone-we see that the total utility is given by

$$
U=\int_{a}^{r_{a}} \phi_{u}(x) d x+\int_{b}^{r_{b}} \phi_{b}(x) d x+\ldots
$$

1 Pareto, G. d. E., August 1892. 
where $a, b, \ldots$ are the values of $x$ that make $\phi_{\alpha}(x), \phi_{b}(x) \ldots$ vanish.

If this is the utility in an increment of time $\Delta t$, then the total utility from time $t_{0}$ to time $t_{1}$ is given by

$$
V=\frac{1}{\Delta t} \int_{t_{0}}^{t_{1}} U d t
$$

If the functions $\phi$ do not change during this time, the different values of $V$ depend upon the values of $r_{a}, r_{b} \ldots$. If there is no saving $r_{a}, r_{b} \ldots$ are known where $p_{a}, p_{b}$ are known, and these are functions of $t$.

If there is saving, but the total quantity saved is the same at the beginning and end of the time, so that there is no increase or decrease in utility caused by variation in the amount saved, we have

$$
\int_{t_{2}}^{t_{1}}\left(r_{a}+p_{b} r_{b}+\ldots\right) d t=0
$$

We want to maximise $\int_{t_{2}}^{t_{1}} U d t$ subject to the above condition. To do this we must make the variation of

$$
\int_{t_{0}}^{t_{1}}\left\{U-\lambda\left(r_{a}+p_{b} r_{b}+\ldots\right)\right\} d t
$$

vanish, where $\lambda$ is an arbitrary constant.

This gives us

$$
\frac{\partial U}{\partial r^{\prime \prime}}+\frac{\partial U}{\partial r_{b}} \cdot \frac{\partial r_{b}}{\partial r_{a}}+\ldots-\lambda\left(1+p_{b} \frac{\partial r_{b}}{\partial r_{a}}+\ldots\right)=0 .
$$

But

$$
\frac{\partial U}{\partial r_{a}}=\phi_{a}\left(r_{a}\right) ; \frac{\partial U}{\partial r_{b}}=\phi_{b}\left(r_{b}\right) ; \ldots
$$

and

whence

$$
\frac{\partial r_{b}}{\partial r_{a}}=p_{b} \frac{\boldsymbol{\phi}_{a}^{\prime}}{\boldsymbol{\phi}_{b}^{\prime}} ; \frac{\partial r_{c}}{\partial r_{a}}=p_{c} \frac{\boldsymbol{\phi}_{a}^{\prime}}{\boldsymbol{\phi}^{\prime}} ; \ldots
$$

$$
\phi_{c}-\lambda+p_{b}^{2}\left(\boldsymbol{\phi}_{c}-\lambda\right) \frac{\boldsymbol{\phi}_{c}^{\prime}}{\boldsymbol{\phi}_{b}^{\prime}}+p_{c}{ }^{\mathrm{s}}\left(\boldsymbol{\phi}_{c}-\lambda\right) \frac{\boldsymbol{\phi}_{u}^{\prime}}{\boldsymbol{\phi}_{b}^{\prime}}+\ldots=0
$$

or

$$
\left(\phi_{a}-\lambda\right)\left\{1+p^{2}{ }_{b} \frac{\phi_{a}^{\prime}}{\phi_{b}^{\prime}}+p_{c}^{2} \frac{\phi_{a}^{\prime}}{\phi_{b}^{\prime}}+\ldots\right\}=0 .
$$

Hence, if $\phi^{\prime}{ }^{\prime}, \phi_{b}^{\prime}, \ldots$ are all of the same sign, we have $\phi_{a}{ }^{\prime}-\lambda=0$; that is the marginal utility of $A$ is constant. This result is interesting, because in general $\phi_{\omega}^{\prime}, \phi_{b}^{\prime}, \ldots$ are all negative, so that we obtain as our condition for maximum utility that the marginal utility of 
money should be constant. And it is clear, from other considerations, that one of the properties of a good money is that its marginal utility is as nearly as possible constant.

To find our constant we have $\phi_{a}=\lambda ; \phi_{b}=p_{b} \lambda ; \ldots$ so that

$$
r_{c}=\psi_{a}(\lambda) ; r_{b}=\psi_{b}\left(p_{b} \lambda\right) ; \ldots
$$

and therefore

$$
\int_{t_{0}}^{t_{1}}\left\{\psi_{a}(\lambda)+p_{b} \psi_{b}\left(p_{b} \lambda\right)+p_{c} \psi_{c}\left(p_{c} \lambda\right)+\ldots\right\}=0
$$

which gives us $\lambda .^{1}$

Still keeping to our supposition that the marginal utility of a commodity is a function merely of that commodity, we have to consider what is the probable form of this function. The hypothesis that has attracted most attention is Bernouilli's, and Prof. Pareto is right in discussing it at considerable length. But as this is primarily a question for psychologists rather than for economists, it would perhaps be rather out of place to give a detailed discussion here -more especially as there seems to be no sufficient reason for supposing that the above supposition is true. But in the particular case of money it is peculiarly important to make some guess at the form of the utility-function for money, and for this purpose Bernouilli's hypothesis seems a fairly good one for moderate amounts, and has, roughly speaking, been used practically in matters of direct taxation until Sir William Harcourt's recent budget. ${ }^{2}$ The hypothesis is as follows: If $U$ is the total utility derived from an amount of money $m$, then $U=U_{0} \log \frac{m}{a}$; where $a$ is the minimum quantity of money on which existence is possible, without the pain being greater than the pleasure, and $U_{0}$ is a constant of utility depending on the individual. If then we take 'equal sacrifice' as our principle of taxation, and assume that $U_{0}$ and $a$ are approximately the same for most individuals, we see that we should levy our income-tax as follows:- Subtract $\& a$ from everybody's income, and then tax the remainder by a fixed percentage. Since $U=U_{0}$ when $m=a e$ ( $e$ being the base of Napierian logarithms, and equal approximately to $2 \cdot 7)$, we see that $U_{0}$ is the amount of utility a man obtains from an amount of money 2.7 times as large as that on which he can just live. For example, if $£ 30$ is the lowest annual income on which it is possible to live, not in misery, then $U_{0}$ is the utility obtained from $£ 81 .^{3}$

1 Pareto, G. d. E., August 1892.

2 The new income-tax in the United States is also based on this principle. The tax is 2 per cent. on a man's income (when 4000 dollars have been subtracted). The English income-tax is more complex.

3 This was pointed out to me by Mr. W. E. Johnson. 
In connection with the question of the final utility of money, we will consider that of the average level of prices. Denote, as before, the prices of $A, B, C \ldots$ by $1, p_{c}, p_{c} \ldots$ and upon a straight $\left.\right|_{D-D^{\prime}} ^{Y} C_{x}^{C^{\prime}}$ line $X Y$, place the points $A, B, C, \ldots$ so that $\overline{A B}=\log p_{b}$; $\overline{A C}=\log p_{c} \ldots$ Suppose that $A, B, C \ldots$ change their positions to $A^{\prime}, B^{\prime}, C^{\prime}, D^{\prime}$, and that $\overline{B C}=\overline{B^{\prime} C^{\prime}}$ and $\overline{C D}=\overline{C^{\prime} D^{\prime}}$, and so on; but that $\overline{A B} \pm \overline{A^{\prime} B^{\prime}}$, then it is usual to assume that $A$ has moved, and that $B, C, D$ have remained stationary. But we have a right to assume this only upon certain assumptions as to the $\dot{a}$ priori probability of the existence of a cause which would make any of the points move (say) from below to above (in our figure). Suppose we find that $A$ has receded from $B, C, D$ upwards. For any point $B$ let $\tau_{b}$ be the $\dot{a}$ priori probability that a cause exists which makes it move, and $\sigma_{b}$ the $\dot{a}$ prior $i$ probability that if this cause exists it makes $B$ move downwards. Then according to our supposition, either $A$ has moved upwards while $B, C, D$ have remained fixed, or $B, C, D$ have moved downwards while $A$ has remained fixed. The probability that the first of these has occurred is-

$$
\pi_{1}=\tau_{a}\left(1-\sigma_{a}\right)\left(1-\tau_{b}\right)\left(1-\tau_{c}\right) \ldots
$$

and that the second has occurred is

$$
\pi_{2}=\left(1-\tau_{u}\right) \tau_{\alpha} \sigma_{b} \tau_{c} \sigma_{c} \ldots
$$

Hence the probability that the change is due merely to a change in $A$ is $\frac{\pi_{1}}{\pi_{1}+\pi_{2}}$; and that it is due to a change in $B, C, D$, not $A$ is $\frac{\pi_{2}}{\pi_{1}+\pi_{2}}$. If we know absolutely nothing about the possible causes, we may take all the probabilities equal to $\frac{1}{2}$; in this case, if $\delta$ is the number of $A, B, C$. . . we obtain

$$
\begin{gathered}
\pi_{1}=\left(\frac{1}{2}\right)^{\delta+1} ; \pi_{2}=\left(\frac{1}{2}\right)^{2 \delta-1}, \\
\frac{\pi_{1}}{\pi_{1}+\pi_{2}}=\frac{2^{\delta-2}}{2^{\delta-2}+1} ; \frac{\pi_{2}}{\pi_{1}+\pi_{2}}=\frac{1}{2^{\delta-2}+1} .
\end{gathered}
$$

If, for example, $\delta=6$, these ratios become $\frac{16}{17}$ and $\frac{1}{17}$, which is in accordance with the opinion given by Cournot, and generally held.

Next suppose that $\tau_{a}$ is the $\dot{a}$ priori probability of a cause which makes $A$ move upwards ; $\tau_{1}$ is the probability that there is a cause which makes $\delta_{1}$ of the points $B, C, D$, move downwards; $\tau_{2}$ the probability of another cause making another $\delta_{2}$ move in the same direction, and so on. We have

$$
\begin{aligned}
& \pi_{1}=\tau_{a}\left(1-\tau_{1}\right)\left(1-\tau_{2}\right) \ldots \\
& \pi_{2}=\left(1-\tau_{a}\right) \tau_{1} \tau_{2} \tau_{3} \cdots
\end{aligned}
$$


If

we obtain

$$
\tau_{a}=\frac{2}{3} ; \tau_{b}=\frac{2}{3} ; \tau_{c}=\frac{2}{3} ; . . .
$$

$$
\pi_{1}=\frac{2}{3^{\epsilon+1}} ; \pi_{2}=\frac{2^{\epsilon}}{3^{\epsilon+1}},
$$

where $\epsilon$ is the number of classes into which the $\delta$ commodities have been divided.

The probability that $A$ has moved is $\frac{1}{1+2^{e-1}}$; and that the other points have moved is $\frac{2^{\epsilon-1}}{1+2^{c-1}}$. If, for example, $\epsilon=4$, these ratios become $\frac{1}{9}, \frac{8}{9} \cdot 1$

This shows us that, if we do happen to know anything about the probable causes, it is most unsafe to accept the common-sense view without further investigation.

The rest of Prof. Pareto's discussion of the variation of the average level prices is somewhat slight, and is disappointing to any one who has read Professor Edgeworth's report to the British Association in 1887 on Index numbers.

Hitherto we have assumed that the marginal utility of a commodity is a function of the amount of that commodity only. But this is clearly not strictly true, and more accurately we must say that the final utility of a commodity $B$, is a function not merely of $r_{b}$ (the amount of $B$ ), but also of $r_{c}, r_{d} \ldots$. (the amounts of $C, D \ldots$ ), so that the total utility, $U$, instead of being

is really given by

$$
\int_{b}^{r_{b}} \phi_{b}(x) d x+\int_{c}^{r_{c}} \phi_{c}(x) d x+\ldots
$$

$$
U=\int_{b}^{r_{b}} \phi_{b}\left(x, r_{c}, r_{l} \ldots\right) d x+\int_{e}^{r_{e}} \phi_{c}\left(x, r_{b}, r_{a} \ldots\right) d x+\ldots
$$

But it seems probable that the total utility derived from the possession of the various amounts of various commodities is a function of the amounts of these commodities possessed so that

and

$$
U=\Phi\left(r_{a}, r_{b}, r_{c} \ldots\right)
$$

$$
\phi_{a}=\frac{\partial U}{\partial r_{a}}, \phi_{b}=\frac{\partial U}{\partial r_{b}},
$$

This suggests some obvious analogies from mechanics-if we take our total utility to correspond to a potential function-and we obtain the same sort of maxima and minima problems in both mechanics and economics.

It is, however, by no means obvious from our equations that a total utility function exists, though from general considerations it

1 Pareto, G. d. E., January 1893. 
seems probable. Suppose, to take the case of only three commodities, that a man possesses amounts $r_{a}, r_{b}, r_{c}$ of $A, B, C$, and that $\phi_{a} \phi_{b} \phi_{c}$ denote the utility of the increments $d r_{a}, d r_{b}, d r_{c} \ldots$ Then under what conditions are the $\phi s$ partial differentials of a certain function $f\left(r_{a}, r_{b}, r_{c}\right)$ of the three variables? If the utility is a maximum, we have

or

$$
\phi_{a c} d r_{a}+\phi_{b} d r_{b}+\phi_{r} d r_{c}=0,
$$

$$
d r_{a}+p_{b} d r_{b}+p_{c} d r_{c}=0 .
$$

If there is a total utility function $f\left(r_{a}, r_{b}, r_{c}\right)$, and this is maximised, we have

$$
\frac{\partial f}{d r_{a}} d r_{a}+\frac{\partial f}{\partial r_{b}} d r_{b}+\frac{\partial f}{\partial r_{c}} d r_{c}=0
$$

These equations are the same if

$$
\frac{\partial f}{\partial r_{a}}=\mu ; \frac{\partial f}{\partial r_{b}}=\mu p_{b} ; \quad \frac{\partial f}{\partial r_{c}}=\mu p_{c} .
$$

Eliminating the $\mu$ s and $f$ we obtain

$$
\frac{\partial p_{b}}{\partial r_{c}}-\frac{\partial p_{c}}{\partial r_{b}}=p_{c} \frac{\partial p_{b}}{\partial r_{a}}-p_{b} \frac{\partial p_{c}}{\partial r_{a}}
$$

which is the condition that a total utility-function exists. ${ }^{1}$ It is not obvious that this equation must necessarily hold. In the case of two variables we see that there must be a total utility-function. It is evidently a matter of some importance to determine whether or no a total utility function must exist; if it does not exist it seems probable that the amount of utility may be capable of indefinite increase.

The last theorem of Sig. Pareto's that will be quoted here, is his proof that, under certain theoretical conditions, maximum utility is attained by free competition.

We use our previous notation, but in addition denote the commo. dities or services necessary for the production of the commodities $A, B, C \ldots$ by $T, S, V, \ldots$ and we suppose that amounts $b_{t}, b_{s}$, $b_{v} \ldots$ of these are required to make a unit of $B, c_{t}, c_{s}, c_{v} \ldots$ to make a unit of $C$, and so on. We denote the prices of these by $p_{t}, p_{s}$, $p_{v} \ldots$ Then the cost of production prices of $A, B, C \ldots$ (denoted by $\pi_{\iota}, \pi_{b}, \pi_{c} \ldots$ ) are given by

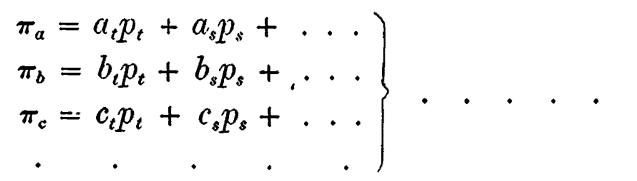

under free competition $p_{a}, p_{b}, p_{c} \ldots$ will approximate to $\pi_{a}, \pi_{b}, \pi_{c} \ldots$

1 Pareto, G. d. E., October 1893. Instead of the equation above given, Sig. Pareto gives $\frac{\partial p_{b}}{\partial r_{c}}=\frac{\partial p_{c}}{\partial r_{b}}$. 
The undertaker wishes to minimise $\pi_{a}, \pi_{b}, \pi_{c} \ldots$ when $p_{t}, p_{s} \ldots$ are given in the market, so that for our present purpose we may suppose them constant.

The coefficients of production for each article are connected by a relation, so that we have

$$
\left.\begin{array}{c}
F_{a}\left(a_{t}, a_{s}, \ldots\right)=0 \\
F_{b}\left(b_{t}, b_{s}, \ldots\right)=0 \\
F_{c}\left(c_{t}, c_{s}, \ldots\right)=0 \\
. \quad . \quad .
\end{array}\right\}
$$

If $m$ is the number of the commodities $A, B, C, \ldots$ and $n$ the number of the commodities and services $T, S, M, \ldots$ we may say that $a_{t}$ is a function of the $\overline{n-1}$ independent variables $a_{s}, a_{v} \ldots ; b_{t}$ is a function of the $\overline{n-1}$ independent variables $b_{s}, b_{v} \ldots$, and so on.

Then differentiating equations (1) to obtain the minimum conditions, we have

$$
\left.\begin{array}{c}
p_{t} \frac{\partial a_{t}}{\partial a_{s}}+p_{s}=0 ; p_{t} \partial a_{t}+p_{v}=0 ; \ldots \\
p_{t}^{\partial b_{t}}+p_{s}=0 ; p_{t} \frac{\partial b_{t}}{\partial b_{v}}+p_{x}=0 ; \ldots
\end{array}\right\} . .
$$

These $m(n-1)$ equations together with the $m$ equations (2), give us the $m n$ equations necessary for the determination of the $m n$ coefficients of production.

Now suppose that $a_{s}$ increase by $d a_{s}$, then the utility enjoyed by an individual, measured in terms of $A$, will increase by

but

$$
\left[\frac{\partial r_{1 a}}{\partial a_{s}}+\frac{\phi_{1 b}}{\phi_{1} a} \cdot \frac{\partial r_{1^{b}}}{\partial a_{s}}+\frac{\phi_{1 c}}{\phi_{1} a} \cdot \frac{\partial r_{1 c}}{\partial a_{s}}+\ldots\right] d a_{s}
$$

$$
\frac{\phi_{1 b}}{\phi_{1 a}}=p_{b} ; \frac{\phi_{1 c}}{\phi_{1 a}}=p_{c} ; \ldots
$$

hence for the entire society the increase in utility measured in terms of $A$ will be

where

$$
\left[\frac{\partial R_{a}}{\partial a_{s}}+p_{b} \frac{\partial R_{b}}{\partial a_{s}}+p_{c} \frac{\partial R_{c}}{\partial a_{s}}+\ldots\right] d a_{s}
$$

$$
\begin{aligned}
& R_{a}=r_{1 a}+r_{2 a}+r_{3 a}+\ldots \\
& R_{b}=r_{1 b}+r_{2 b}+r_{3 b}+\ldots
\end{aligned}
$$

and the total sacrifice again measured in $A$ will increase by

$$
\left[p_{t} \frac{\partial R_{t}}{\partial a_{s}}+p_{s} \frac{\partial R_{s}}{\partial a_{s}}+\ldots\right] d a_{s}
$$


And for maximum utility we obtain the series of equations

$$
\left.\begin{array}{c}
\frac{\partial R_{a}}{\partial a_{s}}+p_{b} \frac{\partial R_{b}}{\partial a_{s}}+\ldots-p_{t} \frac{R_{t}}{\partial a_{s}}-p_{s} \frac{\partial R_{s}}{\partial a_{s}}-\ldots=0 \\
\frac{\partial R_{a}}{\partial b_{s}}+p_{b} \frac{\partial R_{b}}{\partial b_{s}}+\ldots-p_{t} \frac{\partial R_{t}}{\partial b_{s}}-p_{s} \frac{\partial R_{s}}{\partial b_{s}}-\ldots=0
\end{array}\right\} .
$$

These $m(n-1)$ equations together with the $m$ equations (2) give the $m n$ equations necessary to determine the mn coefficients of production.

We have now to show that the coefficients of production determined by free competition have the same values as those obtained if we determine them by the condition of obtaining maximum utility with minimum sacrifice. We have

$$
\left.\begin{array}{l}
R_{t}=a_{t} R_{a}+b_{t} R_{b}+\ldots \\
R_{s}=a_{s} R_{c}+b_{s} R_{b}+\ldots .
\end{array}\right\} . . . . .
$$

Differentiating these we obtain

$$
\begin{aligned}
& \frac{\partial R_{t}}{\partial a_{s}}=R_{u} \frac{\partial a_{t}}{\partial a_{s}}+a_{t} \frac{\partial R_{a}}{\partial a_{s}}+b_{t} \frac{\partial R_{b}}{\partial a_{s}}+\ldots \\
& \frac{\partial R_{s}}{\partial a_{t}}=R_{a}+a_{s} \frac{\partial R_{a}}{\partial a_{s}}+b_{s} \frac{\partial R_{b}}{\partial a_{s}}+\ldots \\
& \frac{\partial R_{v}}{\partial a_{s}}=\quad a_{v} \frac{\partial R_{a}}{\partial a_{s}}+b_{v} \frac{\partial R_{b}}{\partial a_{s}}+\ldots
\end{aligned}
$$

Under free competition $\pi_{a}=p_{a}$, \&c. ; if we multiply the first of the above equations by $p_{t}$, the second by $p_{s}$, and so on; and add them, making use of (2), we obtain

$$
\begin{gathered}
p_{a} \frac{\partial R_{a}}{\partial a_{s}}+p_{b} \partial R_{b}+\ldots-p_{t} \frac{\partial R_{t}}{\partial a_{s}}-p_{s} \frac{\partial R_{s}}{\partial a_{s}}-\ldots \\
=-R_{a}\left(p_{t} \frac{\partial a_{t}}{\partial a_{s}}+p_{s}\right),
\end{gathered}
$$

and by (3) this equals zero.

Thus we obtain the first equation of (4), and we can obtain the rest of equations (4) in a similar manner, which proves the proposition. ${ }^{1}$

Prof. Pareto then proceeds to answer the objection that instead of summing utilities he has only summed amounts of commodities which give these utilities. We do not propose to follow him in the discussion of these difficulties in this place.

Sig. Barone, in his article upon Consumers' Rent, has not made use of mathematical reasoning in the text, but in some mathematical notes he gives the most important portions of his argument.

I Pareto, G. d. E., July 1894. 
For simplicity, we consider only three commodities and money. Suppose that $p_{a}, p_{b}, p_{c}$ are the market prices of the commodities $A, B, C$ when an individual has a quantity of money $M$, whose marginal utility is $m$. And let $\phi_{a}\left(r_{a}\right), \phi_{b}\left(r_{b}\right), \phi_{c}\left(r_{c}\right)$ denote the marginal utilities of amounts $r_{a}$ of $A, r_{b}$ of $B, r_{c}$ of $C$. If the price $p_{a}, p_{b}, p_{c}$ remain constant during the process in which the individual considered obtains and spends his money, then the procedures of Professor Walras and Professor Marshall lead to the same result. But if this is not so, and $p_{a}$ becomes $p_{a}+\Delta p_{a}, p_{b}$ becomes $p_{b}+\Delta_{b}, p_{c}$ becomes $p_{b}+\Delta p_{c}$, then, according to Professor Walras. we obtain

$$
\left.\begin{array}{l}
\frac{\phi_{a}\left(r_{a}+\Delta r_{a}\right)}{p_{a}+\Delta p_{a}}=m+\Delta m \\
\frac{\phi_{b}\left(r_{b}+\Delta r_{b}\right)}{p_{b}+\Delta p_{b}}=m+\Delta m \\
\frac{\phi_{c}\left(r_{c}+\Delta r_{c}\right)}{p_{c}+\Delta p_{c}}=m+\Delta m
\end{array}\right\}
$$

Neglecting small quantities of the second order, we obtain from these

$$
\begin{aligned}
\Delta r_{a} & =\frac{p_{a}}{\phi_{a}^{\prime}\left(r_{a}\right)} \Delta m+\frac{\phi_{a}\left(r_{a}\right)}{p_{a} \phi_{a}^{\prime}\left(r_{a}\right)} \Delta p_{a} \\
\Delta r_{b} & =\frac{p_{b}}{\phi_{b}^{\prime}\left(r_{b}\right)} \Delta m+\frac{\phi_{b}\left(r_{b}\right)}{p_{b} \phi_{b}^{\prime}\left(r_{b}\right)} \Delta p_{b} \\
\Delta r_{c} & =\frac{p_{c}}{\phi_{c}^{\prime}\left(r_{c}\right)} \Delta m+\frac{\phi_{c}\left(r_{c}\right)}{p_{c} \phi_{c}^{\prime}\left(r_{c}\right)} \Delta p_{c}
\end{aligned}
$$

Professor Marshall assumes that $m$ remains constant, and consequently his increments (which we denote by $\delta r_{a}, \delta r_{b}, \delta r_{c}$ ) are

$$
\left.\begin{array}{rl}
\delta r_{a} & =\frac{\phi_{a}\left(r_{a}\right)}{p_{a} \phi_{a}^{\prime}\left(r_{a}\right)} \Delta p_{a} \\
\delta r_{b} & =\frac{\phi_{b}\left(r_{b}\right)}{p_{b} \phi_{b}^{\prime}\left(r_{b}\right)} \Delta p_{b} \\
\delta r_{e} & =\frac{\phi_{c}\left(r_{c}\right)}{r_{c} \phi_{c}^{\prime}\left(r_{c}\right)} \Delta p_{c}
\end{array}\right\}
$$

According to Professor Walras the individual spends an amount of money $M$, while Professor Marshall makes him spend $M+\delta M$ where $\delta M$ is evidently given by

$$
\delta M=r_{a} \Delta p_{a}+p_{a} \delta r_{c}+r_{b} \Delta p_{b}+p_{b} \delta r_{b}+r_{c} \Delta p_{c}+p_{c} \delta r_{c} .
$$

If then $R_{w}$ and $R_{u}$ denote the consumer's rent, according to the two writers we have

$$
\begin{aligned}
& R_{w}=U+\Delta U-m . M \\
& R_{m}=\delta U+U-m(M+\delta M) \\
\therefore R_{w} & =R_{m}=\Delta U-\delta U+m \delta . M .
\end{aligned}
$$


But

$$
\begin{gathered}
\Delta U=\phi_{a}\left(r_{a}\right) \Delta r_{a}+\phi_{b}\left(r_{b}\right) \Delta r_{b}+\phi_{c}\left(r_{c}\right) \Delta r_{c}, \\
\delta U=\phi_{a}\left(r_{a}\right) \delta r_{a}+\phi_{b}\left(r_{b}\right) \delta r_{b}+\phi_{c}\left(r_{c}\right) \delta r_{c} ; \\
\therefore \Delta U-\delta U=\phi_{a}\left(r_{a}\right)\left\{\Delta r_{a}-\delta r_{a}\right\}+\phi_{b}\left(r_{b}\right)\left\{\Delta r_{b}-\delta r_{b}\right\}+\phi_{c}\left(r_{c}\right)\left\{\Delta r_{c}-\delta r_{c}\right\} .
\end{gathered}
$$

And, also,

$$
\begin{aligned}
& r_{a} \Delta p_{a}+p_{a} \Delta r_{a}+r_{b} \Delta p_{b}+p_{b} \Delta r_{b}+r_{c} \Delta p_{c}+p_{c} \Delta r_{c}=0, \\
& r_{a} \Delta p_{a}+p_{a} \delta r_{a}+r_{b} \Delta p_{b}+p_{b} \delta r_{b}+r_{c} \Delta p_{c}+p_{c} \delta r_{c}=\delta M,
\end{aligned}
$$

Hence, by subtraction,

$$
p_{a}\left\{\Delta r_{a}-\delta r_{a}\right\}+p_{b}\left\{\Delta r_{b}-\delta r_{b}\right\}+p_{c}\left\{\Delta r_{c}-\delta r_{c}=-\delta M,\right.
$$

or

$$
\begin{aligned}
& \phi_{a}\left(r_{a}\right)\left\{\Delta r_{a}-\delta r_{a}\right\}+\phi_{b}\left(r_{b}\right)\left\{\Delta r_{b}-\delta r_{b}\right\}+\phi_{c}\left(r_{c}\right)\left\{\Delta r_{c}-\delta r_{c}\right\}=-m \delta M ; \\
& \therefore \Delta U-\delta U=-m \delta M .
\end{aligned}
$$

Hence, $R_{w}-R_{m}=0$ to the second order of small quantities. ${ }^{1}$

This result is in accordance with the statement on page 753 of the Principles of Economics.

Another, and perhaps better proof of this is given by Sig. Barone in his next article. He then proceeds to discuss what happens when a state of equilibrium is disturbed. Keeping to our previous notation, but for simplicity limiting the number of individuals of the commodities $A, B$, and of the commodities and services $T, S$, to two each, we have in equilibrium

$$
\left.\begin{array}{l}
\frac{\phi_{1 a}\left(r_{1 a}\right)}{p_{a}}=\frac{\phi_{b}\left(r_{1 b}\right)}{p_{b}}=\frac{\phi_{1 t}\left(r_{1 t}\right)}{p_{t}}=\frac{\phi_{1 s}\left(r_{1 s}\right)}{p_{s}} \\
\frac{\phi_{2 a}\left(r_{2 a}\right)}{p_{1 a}}=\frac{\phi_{2 b}\left(r_{2 b}\right)}{p_{b}}=\frac{\phi_{2 t}\left(r_{2 t}\right)}{p_{t}}=\frac{\phi_{2 s}\left(r_{2 s}\right)}{p_{s}}
\end{array}\right\}
$$

Since the marginal utilities of the products demanded and the services offered must, for each individual, be proportional to the prices, we also have:-

$$
\left.\begin{array}{l}
p_{a} r_{1 a}+p_{b} r_{1 b}=p_{t} r_{1 t}+p_{s} r_{1 s} \\
p_{a} r_{2 a}+p_{b} r_{2 b}=p_{t} r_{2 t}+p_{s} r_{2 s}
\end{array}\right\}
$$

Further, if $t_{a}, t_{b}$ denote the coefficients of production of $A, s_{a}, s_{b}$ those of $B$, we have

$$
\left.\begin{array}{r}
r_{1 t}+r_{2 t}=t_{a}\left(r_{1 a}+r_{2 a}\right)+t_{b}\left(r_{1 b}+r_{2 b}\right) \\
r_{1 s}+r_{2 s}=s_{a}\left(r_{1 a}+r_{2^{a}}\right)+s_{b}\left(r_{1 b}+r_{2 b}\right)
\end{array}\right\}
$$

' Barone, G. d. E., September 1894. 
If now the equilibrium is disturbed by a $\operatorname{tax} r$ on the commodity $A$ we have

$$
\delta r_{1 a}=\frac{\phi_{1 a}\left(r_{1 a}\right)}{p_{a} \phi_{1 a}^{\prime}\left(r_{1 a}\right)} \delta p_{a}
$$

and similar equations in place of (1).

Summing up the individual changes in demand and supply, and letting $\delta D_{a}, \delta D_{b}$ denote the variations of the total demand for $A$ and $B$; and $\delta O_{t}, \delta O_{s}$, the variations of the total supply of $T$ and $S$, we obtain

$$
\delta D_{a}=c_{a} p_{a} ; \delta D_{b}=c_{b} \delta p_{b} ; \delta O_{t}=c_{t} \delta p_{t} ; \delta O_{s}=c_{s} \delta p_{s} .
$$

The equations (3) now become

$$
\left.\begin{array}{l}
\delta O_{t}=t_{a} \delta D_{a}+s_{b} \delta D_{b} \\
\delta O_{s}=s_{a} \delta D_{a}+s_{b} \delta D_{b}
\end{array}\right\}
$$

The equations (4) become

$$
\left.\begin{array}{l}
\delta p_{u}=r+t_{u} \delta p_{t}+s_{u} \delta p_{s} \\
\delta p_{b}=\begin{array}{rl}
t_{b} \delta p_{t}+s_{b} \delta p_{s}
\end{array}
\end{array}\right\}
$$

from these and from

$$
\left.\begin{array}{l}
\delta p_{t}=t_{u}{ }_{c_{t}}^{c_{a}} \delta p_{a}+t_{b}^{c_{b}} \delta p_{b} \\
\delta p_{s}=s_{a} \frac{c_{a}}{c_{s}} \delta p_{a}+s_{b} \frac{c_{b}}{c_{s}} \delta p_{b}
\end{array}\right\}
$$

we can approximate to the values of the unknowns.

Put

then

$$
\delta p_{t}=0, \delta p_{s}=0 ;
$$

whence

$$
\delta p_{a}=r, \delta p_{b}=0
$$

$$
\delta p_{t}=t_{a}^{\frac{c_{a}}{c_{t}} ; \delta p_{s}}=s_{c_{s}}^{c_{a}} r ;
$$

then putting these values in the equations, which give $\delta p_{a}$ and $\delta p_{b}$, and proceeding in this way, we can obtain our unknowns to any degree of approximation. ${ }^{1}$

At this point we will stop. We have been told that 'it seems doubtful whether any one spends his time well in reading lengthy translations of economic doctrines into mathematics that have not been made by himself.' This is certainly true if the translations are lengthy; but the two economists from whose writings this paper is taken are not very lengthy in their exposition, though perhaps they are rather too fond of an imposing array of mathematical symbols. So large a proportion of mathematical economics is either bad or dull, or

${ }^{3}$ Barone, G. d. E., November 1894. 
both dull and bad, that it is a pleasure to find so much that is good and also by no means unimportant, in a rather scattered series of articles by two writers during the past few years. The time seems to have come when a book on the pure theory of economics would be of great use to the student. Such a book could contain a great deal of accurate reasoning within a small compass, so long as the writer was not afraid of freely using mathematical methods. Professor Pantaleoni's book is too elementary ; ${ }^{1}$ the mathematical appendix to the Principles of Economics is too short. Until a book appears, the student has to pick out his pure theory from a number of books and review articles written in different languages. The object of this article has been merely to aid him in this process of selection, without making any serious attempt either to criticise or to add to what has up to the present been done on the subject.

\section{P. ȘANGER}

\section{Capitalists and Landowners in Italy.}

THe financial scheme which was laid before the Italian Chamber of Deputies and Senate in 1894 is not only of interest to the economist, but also offers an ample field for discussion to the student of sociology.

The facts were as follows: There was a deficit of several tens of millions of lire, and it was by no means easy to discover new sources of taxation to supply this money. For, when a nation has reached a certain stage of saturation in the matter of taxes, new ones merely tend to sterilise still further the previous taxes, and the useful effect of the fresh ones is in great measure lost.

It is said that there is a lack of equilibrium between the financial needs and the national income, and a state of neediness is so usual that the sacrifice the nation has to undergo to support it reaches an oppressive limit. Now this can only be permitted in case of any temporary necessity in which the State happens to be. ${ }^{2}$ Whenever these conditions are absent and the circumstances under consideration are of some duration, theory forbids the attainment of this oppressive limit. But Wagner judiciously remarks that it is not always so in practice, and that the interested parties, namely, the statesmen at the head of the Government, who make a boast of their empiricism, make no attempt to harmonise their proposals with theory. They lay stress on the increase of expenditure, and go on to say, 'In one way or other this expenditure has to be met, and as money is wanted we must find it.' Save for acting with scant honesty when the interest on the Public Funds is payable, for then they take

1 Principii di Economia pura, per Maffeo Pantaleoni.

2 Compare the condition of Russia in 1812-13, of Prussia 1813-14, and of the United States during the War of Secession. 